\title{
Clinical significance of the expression of long non-coding RNA HOTAIR in primary hepatocellular carcinoma
}

\author{
MASAHISA ISHIBASHI ${ }^{1,2}$, RYUNOSUKE KOGO ${ }^{1}$, KOHEI SHIBATA ${ }^{1}$, GENTA SAWADA ${ }^{1}$, \\ YUSUKE TAKAHASHI $^{1}$, JUNJI KURASHIGE ${ }^{1}$, SAYURI AKIYOSHI $^{1}$, SHIN SASAKI $^{3}$, TAKESHI IWAYA ${ }^{1,2}$, \\ TOMOYA SUDO ${ }^{1}$, KEISHI SUGIMACHI ${ }^{1}$, KOSHI MIMORI $^{1 *}$, GO WAKABAYASHI $^{2}$ and MASAKI MORI ${ }^{4 *}$ \\ ${ }^{1}$ Department of Surgery, Kyushu University Beppu Hospital, Beppu, Oita 874-0838; ${ }^{2}$ Department of Surgery, \\ Iwate Medical University, Morioka, Iwate $020-8505 ;{ }^{3}$ Omori Red Cross Hospital, Oota, Tokyo 143-8527; \\ ${ }^{4}$ Department of Gastroenterological Surgery, Osaka University, Suita, Osaka 565-0871, Japan
}

Received October 8, 2012; Accepted November 16, 2012

DOI: $10.3892 /$ or.2012.2219

\begin{abstract}
The functions of many long non-coding RNAs (ncRNAs) in human cancers have not yet been elucidated. The long ncRNA HOTAIR is expressed from the developmental HOXC locus located on chromosome 12q13.13. Previous reports have demonstrated that HOTAIR associates with chromatin modifications in cooperation with the Polycomb complex PRC2, and promotes breast and colorectal cancer metastasis. In this study, we examined the clinical significance of HOTAIR expression in patients with hepatocellular carcinoma (HCC). HOTAIR expression was detected in primary HCCs in 13 out of 64 patients. Patients with HOTAIR expression had significantly poorer prognoses and a larger primary tumor size than those without HOTAIR expression, similar to studies in breast and colorectal cancers. Moreover, introduction of human HOTAIR into liver cancer cells revealed that HOTAIR promoted more rapid proliferation compared to control cells. Thus, although the clinical significance of HOTAIR expression in HCC may not be as pronounced as that in breast and colorectal cancers, the current study demonstrates that HOTAIR expression is associated with HCC progression, warranting further studies.
\end{abstract}

\section{Introduction}

Recently, many studies have focused on investigating the role of non-coding RNAs (ncRNAs), RNA sequences that are not translated into protein. Not only are the functions of ncRNAs only partially understood, but the clinical or biological significance of most ncRNAs has not yet been determined.

Correspondence to: Professor Koshi Mimori, Department of Surgery, Kyushu University, Beppu Hospital, 4546 Tsurumi, Beppu, Oita 874-0838, Japan

E-mail: kmimori@beppu.kyushu-u.ac.jp

*Contributed equally

Key words: non-coding RNA, HOTAIR, hepatocellular carcinoma
Among ncRNAs, long ncRNAs, consisting of over 200 bases have been reported to associate with DNA-binding proteins, such as chromatin modifying complexes, and certain ncRNAs can epigenetically regulate the expression of multiple genes through this mechanism (1-3).

Hox transcript antisense intergenic RNA (HOTAIR) is a long ncRNA that was identified from a custom tilling array of the HOXC locus (12q13.13) (4). HOTAIR forms a complex with the polycomb-repressive complex 2 (PRC2), composed of EZH2, SUZ12 and EED, to trimethylate histone $\mathrm{H} 3$ at lysine 27 (H3K27me3), thereby inhibiting HOXD gene expression. Thus, HOTAIR epigenetically regulates the expression of $H O X D$, a gene located on a separate chromosome. Recent studies have demonstrated the clinical significance of HOTAIR in surgical solid malignancies. Gupta et al found that patients with high HOTAIR expression in primary lesions had a poorer prognosis for both overall- and metastases-free survival than those with low HOTAIR expression in breast cancer (4). Moreover, we reported the clinical significance of HOTAIR in patients with advanced colorectal cancer (CRC), where HOTAIR was more highly expressed in cancerous tissues than in noncancerous tissues (5). High HOTAIR expression correlated well with the presence of liver metastases, and was significantly associated with poorer prognoses. In addition, we found that HOTAIR expression was associated with a genome-wide reprogramming of PRC2 (SUZ12, EZH2 and H3K27me3) by gene set enrichment analysis using cDNA array data from a pilot study (5). Since HOTAIR expression has clinical significance in both breast and colorectal cancers, we expect that this ncRNA may also play an important role in other intractable malignancies.

Hepatocellular carcinoma (HCC) is one of the most common cancers in the world. In Japan, HCC is an intractable tumor, causing approximately 30,000 deaths per year and representing the third leading cause of death from malignant neoplasms in men, and the fifth leading cause of death from malignant neoplasms in women (6,7). The major causes of HCC are viral infections, alcohol and tobacco use. While efforts have been made to identify appropriate prognostic markers for HCC (8-12), including primary tumor size, elevated AFP levels, and gene expression markers in the primary tumor, these method have not proven adequate to predict the prognosis 
of all HCC patients. In addition, few studies describing the expression of ncRNAs and their clinical significance in HCC have been published. In the present study, we determined the clinicopathological significance of HOTAIR expression in HCC patients, and investigated whether HOTAIR is a useful prognostic indicator in HCC patients.

\section{Materials and methods}

Clinical tissue samples. A total of 64 patients with HCC who underwent surgery at Beppu Hospital were enrolled in this study. The resected tumor and paired non-tumor tissue specimens were immediately frozen in liquid nitrogen and kept at $-80^{\circ} \mathrm{C}$ until analysis. Frozen tissue specimens were homogenized in guanidinium thiocyanate, and total RNAs were obtained by ultracentrifugation through a cesium chloride cushion. Written informed consent was obtained from all patients. All patients were closely followed after surgery at regular one-month intervals.

RNA preparation, reverse transcription and quantitative realtime PCR. Total RNA from frozen HCC samples $(\mathrm{n}=64)$ was extracted using Isogen (Nippon Gene Co., Ltd.) following the manufacturer's protocol. As previously reported, cDNAs from all samples were synthesized from $8.0 \mu \mathrm{g}$ of total RNA (13). HOTAIR levels were quantified using a LightCycler 480 Probes Master kit (Roche Applied Science) following the manufacturer's protocol with the following specific HOTAIR primers: (forward, 5'-CAGTGGGGAACTCTGACTCG-3' and reverse, 5'-GTGCCTGGTGCTCTCTTACC-3'). HOTAIR levels were normalized to GAPDH (forward, 5'-GTCAACGGATTTGG TCTGTATT-3' and reverse, 5'-AGTCTTCTGGGTGGCAGT GAT-3').

Cell lines. HepG 2 cells, human liver cancer cells, were provided by the Cell Resource Center for Biomedical Research, Institute of Development, Aging and Cancer, Tohoku University, Japan. All cell lines were maintained in Dulbecco's modified Eagle's media (DMEM) supplemented with $10 \%$ fetal calf serum and antibiotics. We cultured the cells at $37^{\circ} \mathrm{C}$ in a humidified atmosphere of $5 \% \mathrm{CO}_{2}$ and $95 \%$ air.

HOTAIR expression lentiviral vector. To generate a HOTAIR expression lentiviral vector, we amplified full-length human HOTAIR by PCR using MCF7 cDNA. Lentiviruses were produced by transient transfection of HEK293T cells with pCMV-VSV-G-RSV-Rev, pCAG-HIVgp, and either CSII-CMV-HOTAIR or CSII-CMV-MCS (empty) plasmid DNAs (5'-XhoI and 3'-NotI sites) using Lipofectamine 2000 (Invitrogen), following the manufacturer's protocol. Fortyeight hours after cotransfection, the lentivirus-containing supernatant was collected and passed through a $0.45-\mu \mathrm{m}$ filter. The titer of the lentivirus vector in filtered supernatants was estimated by measuring the concentration of HIV p24 gag antigen with an ELISA kit (Perkin-Elmer Life Science).

Cell proliferation assay. Cell proliferation was assessed by 3-(4,5-dimethylthiazol-2-yl)-2,5-diphenyl tetrazolium bromide (MTT) assay. In brief, we plated HepG2 cells infected with either the human HOTAIR full-length lentiviral vector or the empty lentiviral vector, in 96 -well tissue culture plates at a density of $5.0 \times 10^{3}$ cells per well. At different time points $(24,72$, and $120 \mathrm{~h}$ after plating, representing the 0-, 48-, and 96-h time points respectively), $10 \mu \mathrm{l}$ MTT ( $5 \mathrm{mg} / \mathrm{ml}$ in phosphate-buffered saline) was added to each well, and plates were incubated for an additional $4 \mathrm{~h}$ at $37^{\circ} \mathrm{C}$. The colored formazan product was then dissolved in $100 \mu \mathrm{l}$ DMSO. We then evaluated mitochondrial activity, reflecting cellular growth and viability, by measuring the optical density at a test wavelength of 570-650 nm using a microplate reader (Bio-Rad, Japan); results were expressed as optimal density per milligram protein (OD/mg protein).

Statistical analysis. The significance of differences between two groups was estimated using the Student's t-test and the $\chi^{2}$ test. Overall survival curves were plotted according to the Kaplan-Meier method, with the log-rank test applied for comparison. Variables with a p-value of $<0.05$ by univariate analysis were used in subsequent multivariate analysis on the basis of the Cox proportional hazards model. All differences were considered statistically significant when $\mathrm{p}<0.05$. Statistical analyses were conducted using JMP 5 software (SAS Institute).

Gene set enrichment analysis (GSEA) of HCC in the GEO database. For GSEA (14), we used GSE27462 in the GEO database; this was a dataset for RNA expression profiles collected using a genome tiling array in $10 \mathrm{HCC}$ samples (15). HOTAIR expression was treated as a binary variable divided into low or high expression according to medians. For functional gene sets for GSEA, we used gene sets arising from global occupancy of H3K27me3, and EZH2, a PRC2 subunit induced by HOTAIR overexpression in MDA-MB-231 breast cancer cells (4). As a metric for ranking genes in GSEA, the difference between the means of samples with low and high HOTAIR expression was used, and other parameters were set to default values.

\section{Results}

We first evaluated HOTAIR expression in primary tumors from HCC patients $(n=64)$ by quantitative real-time PCR. From these data, we divided the 64 patients with $\mathrm{HCC}$ into a HOTAIR high expression group $(\mathrm{n}=13)$ and a low expression group ( $\mathrm{n}=51)$ (Fig. 1A). In consideration of clinical applications, we set cut-off values as the upper limit of normal samples, and we found a HOTAIR/GAPDH ratio of 0.027 in HCC samples. Patients with high HOTAIR expression had a significantly poorer prognosis with regard to overall survival and a significantly larger tumor size than those with low HOTAIR expression ( $\mathrm{p}<0.01)$ (Fig. 1B). Clinicopathological factors were analyzed between groups (Table I), and no significant differences in other clinicopathological factors or in recurrence-free survival were noted between the high and low expression groups (data not shown).

Subsequently, we examined whether HOTAIR overexpression in HepG2 cells induced more rapid cell growth using an MTT assay. While we observed no significant differences between normal HepG2 cells (control) and HepG2 cells overexpressing HOTAIR, there was a tendency for the HOTAIR-expressing HepG2 cells to grow more rapidly than the control HepG2 cells ( $\mathrm{p}=0.10$ ) (Fig. 2). 
A

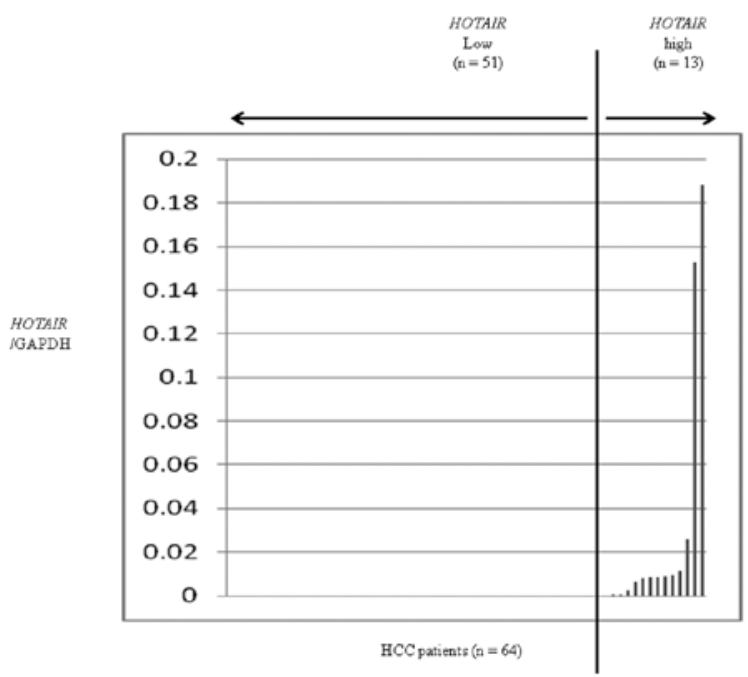

B

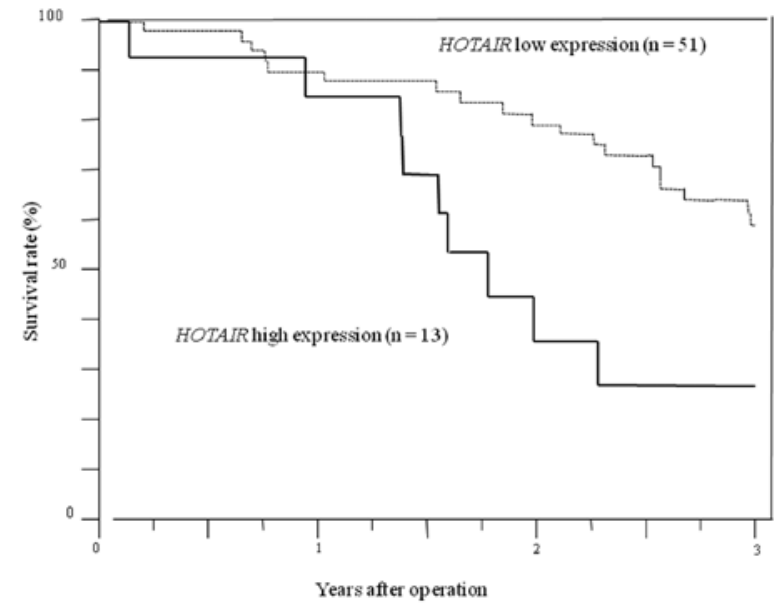

Figure 1. (A) Quantitative real-time PCR analysis of HOTAIR in 64 HCC tissues and the classification based on the HOTAIR level (HOTAIR/GAPDH of 0.0027). Vertical line indicates the borderline of HOTAIR high $(\mathrm{n}=13)$ or low $(\mathrm{n}=51)$ expression. (B) Kaplan-Meier survival curves for HCC patients with HOTAIR high expression $(\mathrm{n}=13)$ and those with low expression $(\mathrm{n}=51)$. The HOTAIR expression group had poorer survival ( $\mathrm{p}<0.01$, Wilcoxon and log-rank test).

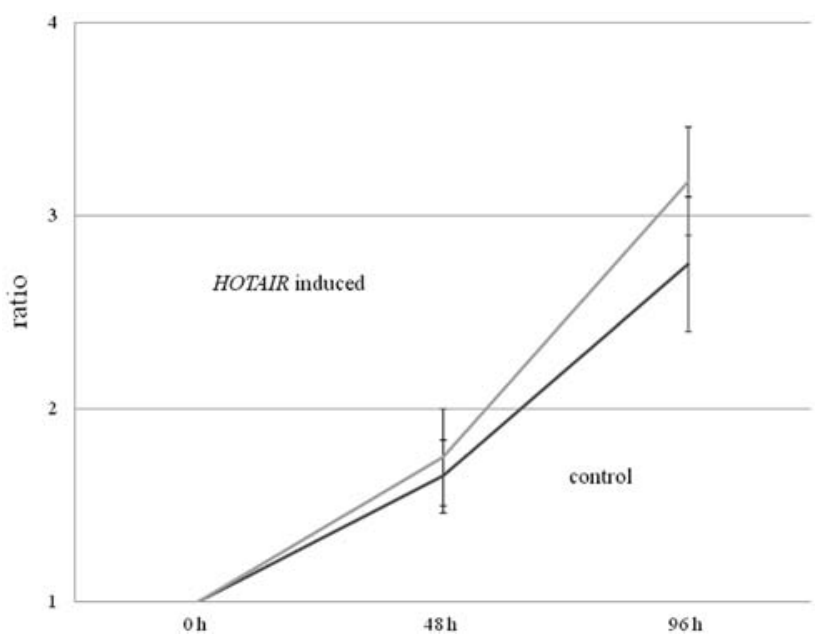

Figure 2. MTT assay of the HepG2 cell line, HOTAIR-expressing group and control group. The figure is plotted using the ratio of each data value to the average of $0 \mathrm{~h} \mathrm{OD} / \mathrm{mg}$ protein. The HOTAIR-induced group tended to growth more rapidly than the control group, but there were no significant differences between the two groups $(\mathrm{p}=0.09)$.
Table I. HOTAIR expression and clinicopathological features of the HCC patients.

\begin{tabular}{lccc}
\hline Features & $\begin{array}{c}\text { HOTAIR } \\
\text { high expression } \\
(\mathrm{n}=13)\end{array}$ & $\begin{array}{c}\text { HOTAIR } \\
\text { low expression } \\
(\mathrm{n}=51)\end{array}$ & p-value \\
\hline Age (years) & $61.3 \pm 2.8$ & $67.2 \pm 1.4$ & 0.06 \\
Gender & & & \\
Male & $9(69.2 \%)$ & $34(66.7 \%)$ & 0.86 \\
Female & $4(30.8 \%)$ & $17(33.3 \%)$ & \\
Virus & & & \\
HBV(+) & $4(30.8 \%)$ & $9(17.7 \%)$ & 0.38 \\
HCV(+) & $9(69.2 \%)$ & $38(74.5 \%)$ & \\
non-B, non-C & $0(0.0 \%)$ & $4(7.8 \%)$ & \\
Child-Pugh & & & \\
A & $11(84.6 \%)$ & $44(86.2 \%)$ & 0.88 \\
B & $2(15.4 \%)$ & $6(11.8 \%)$ & \\
C & $0(0.0 \%)$ & $1(2.0 \%)$ & \\
& & &
\end{tabular}

Tumor size $(\mathrm{cm})$

$5.7 \pm 0.8$

$3.2 \pm 0.4$

$0.009^{\mathrm{a}}$ fc
$(+)$

$(-)$

fc-inf

$(+)$

$(-)$

$(53.9 \%)$

$6(46.1 \%)$

$22(43.1 \%)$

$29(56.9 \%)$

$5(38.5 \%)$

$24(47.1 \%)$

0.61

$7(53.9 \%)$

$20(39.2 \%)$

$1 \quad(7.6 \%)$

$7(13.7 \%)$

$12(92.3 \%)$

$49(96.1 \%)$

$2(3.9 \%)$

b

0

1

im

0

1

2

Tumor number

$\begin{array}{lll}\text { Single } & 9(69.2 \%) & 36(70.6 \%) \\ \text { Multiple } & 4(30.8 \%) & 15(29.4 \%)\end{array}$

$0 \quad(0.0 \%)$

$1(2.0 \%)$

Multiple

$4(30.8 \%)$
0.92

Clinicopathological data were categorized based on HOTAIR high expression or HOTAIR low expression. Specimens from 64 patients with HCC were used for the study. HCC, hepatocellular carcinoma; $\mathrm{HBV}$, hepatitis B surface antigen; HCV, hepatitis C virus; fc, capsular formation; vp, invasion to portal vein; vv, microscopic hepatic vein invasion; b, invasion to bile duct; im, intrahepatic metastasis. ${ }^{\text {aStatis- }}$ tically significant difference. 


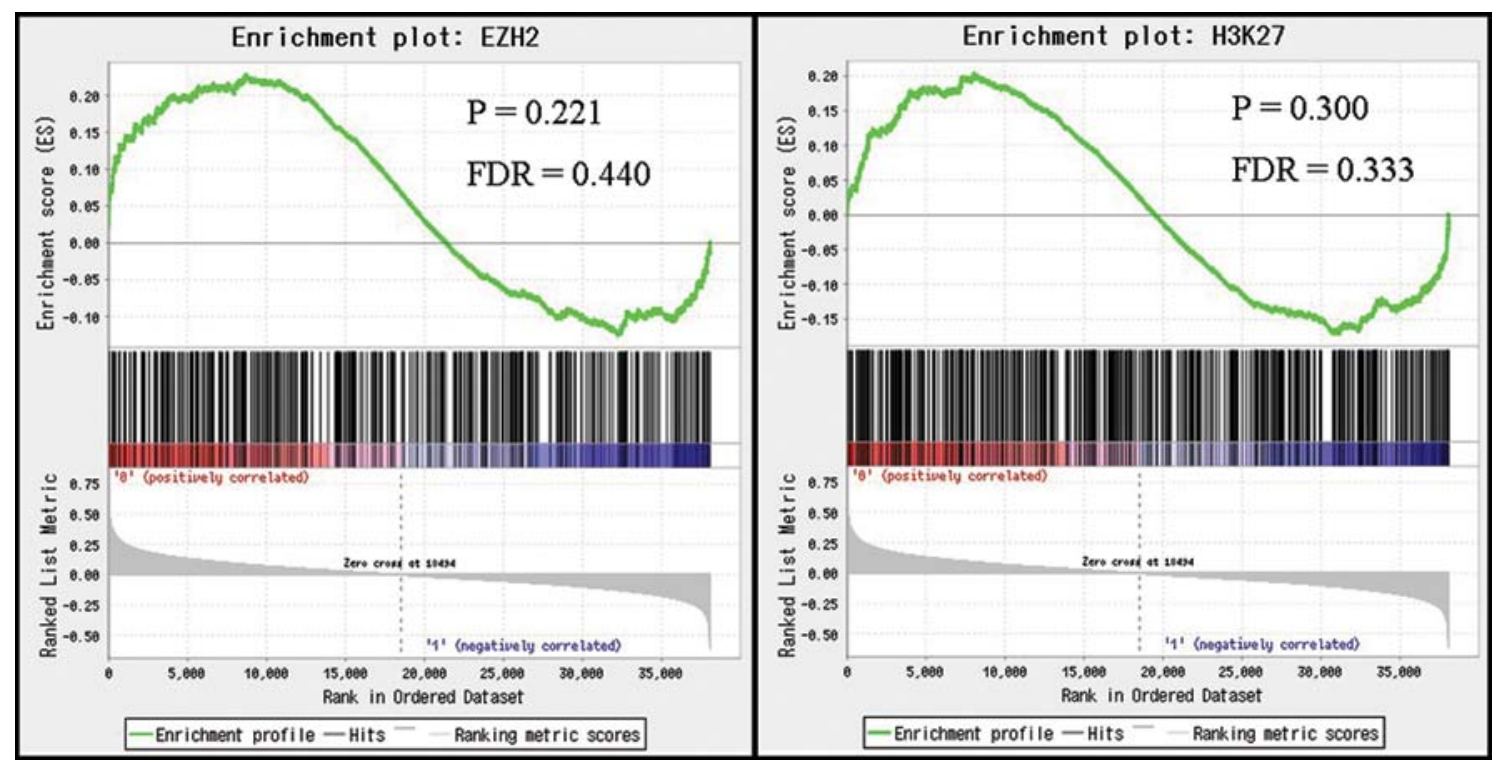

Figure 3. Enrichment plots of gene expression signatures of HOTAIR induced EZH2 and H3K27me3 occupancy sorted according to the differences between the means of samples with high and low HOTAIR expression in GSE27462. The vertical axis in the upper graph indicates Enrichment Score (ES) for the genes in each gene set. The barcode plot indicates the position of the genes in each gene set; red and blue colors represent positive and negative Pearson correlation with HOTAIR expression, respectively. The vertical axis in the lower graph indicates Ranked List Metric. HOTAIR expression levels in HCC were not enriched for these gene sets, as HOTAIR overexpression induced localization of H3K27, and PRC2 subunits, EZH2, in MDA-MB-231 breast cancer cells.

We next investigated the relationship between HOTAIR expression and gene signatures after HOTAIR-induced H3K27me3 and EZH2 occupancy using GSEA. Based on the results of GSEA, there were no significant correlations between HOTAIR expression levels in HCC and the expression levels of signatures induced by $\mathrm{H} 3 \mathrm{~K} 27 \mathrm{me} 3$ and EZH2 occupancy (Fig. 3). These results suggest that HOTAIR expression in HCC may not induce genome-wide retargeting of PRC2, unlike that noted in breast and colorectal cancers.

\section{Discussion}

In the present study, we found that high HOTAIR expression in HCC primary tumors was associated with a poor prognosis. As for clinicopathological factors, there was a significant association between HOTAIR expression and tumor size. Additionally, we found that introduction of human HOTAIR in liver cancer cells resulted in a tendency for more rapid proliferation, compared with the control cells. We speculate that HOTAIR may promote cell growth, explaining the significant association between HOTAIR expression and tumor size in $\mathrm{HCC}$, and leading to poorer prognoses in $\mathrm{HCC}$ patients with high HOTAIR expression.

Recently, researchers have been searching for novel biomarkers to predict tumor recurrence in patients who have undergone liver transplantation (16). In the present study, we showed that high HOTAIR expression in primary HCC could be a useful marker for predicting $\mathrm{HCC}$ recurrence since $\mathrm{HCC}$ cases can be clearly categorized into high and low expression groups.

In previous studies, including ours, HOTAIR expression was shown to interact with multiple genes in cooperation with PRC2 (5), suggesting an important role for HOTAIR in tumor growth and tumorigenicity of breast and colorectal cancers. In addition, Yang et al reported that long ncRNAs, including HOTAIR, are expressed at high levels in HCC tumors in vivo (15). HOTAIR and other long ncRNAs, such as XIST, and other unidentified ncRNAs, may play significant roles in tumor growth in cooperation with PRC2 and histone modification genes in HCC (17-19). In the present study, however, GSEA results showed that the expression profiles generally induced by H3K27me3 and EZH2, a PRC2 element, were not significantly associated with HOTAIR expression in HCC. Therefore, this ncRNA may cooperate with other molecules or in different pathways in HCC, rather than functional with PRC2 complex proteins, and further research is required to fully elucidate these mechanisms.

In conclusion, certain HCC patients express the long ncRNA HOTAIR, and HCC patients with high HOTAIR expression exhibits poorer prognoses than those with low HOTAIR expression. HOTAIR expression in primary HCC tumors may be a prognostic marker in HCC; however, the clinical significance of HOTAIR expression in HCC appears to be less important than that in breast and colorectal cancers, including parameters such as histological grade, tumor depth and lymph node metastasis. Our data demonstrate the need for further study to support the use of ncRNAs as new clinical indicators of poor prognosis in $\mathrm{HCC}$.

\section{Acknowledgements}

The authors thank T. Shimooka, and M. Kasagi for their technical assistance and H. Miyoshi (RIKEN BioResource Center) for providing the lentiviral vector plasmid DNA. This study was supported in part by the following grants and foundations: CREST, Japan Science and Technology Agency (JST); Japan Society for the Promotion of Science (JSPS) Grant-in-Aid for Scientific Research (grant nos. 20390360, 20591547, 20790960, 21591644, 21791295, 21791297, 215921014 and 21679006); the Funding Program for Next Generation World-Leading 
Researchers (LS094); NEDO (New Energy and Industrial Technology Development Organization) Technological Development for Chromosome Analysis; and Grant-in-Aid from the Tokyo Biochemical Research Foundation.

\section{References}

1. Ponting CP, Oliver PL and Reik W: Evolution and functions of long noncoding RNAs. Cell 136: 629-641, 2009.

2. Rinn JL, Kertesz M, Wang JK, et al: Functional demarcation of active and silent chromatin domains in human HOX loci by noncoding RNAs. Cell 129: 1311-1323, 2007.

3. Khalil AM, Guttman M, Huarte M, et al: Many human large intergenic noncoding RNAs associate with chromatin-modifying complexes and affect gene expression. Proc Natl Acad Sci USA 106: 11667-11672, 2009.

4. Gupta RA, Shah N, Wang KC, et al: Long non-coding RNA HOTAIR reprograms chromatin state to promote cancer metastasis. Nature 464: 1071-1076, 2010.

5. Kogo R, Shimamura T, Mimori K, et al: Long noncoding RNA HOTAIR regulates polycomb-dependent chromatin modification and is associated with poor prognosis in colorectal cancers. Cancer Res 71: 6320-6326, 2011.

6. Kudo M, Izumi N, Kokudo N, et al: Management of hepatocellular carcinoma in Japan: Consensus-Based Clinical Practice Guidelines proposed by the Japan Society of Hepatology (JSH) 2010 updated version. Dig Dis 29: 339-364, 2011.

7. Umemura T, Ichijo T, Yoshizawa K, Tanaka E and Kiyosawa K: Epidemiology of hepatocellular carcinoma in Japan. J Gastroenterol 44 (Suppl 19): 102-107, 2009.

8. Ammerpohl O, Pratschke J, Schafmayer C, et al: Distinct DNA methylation patterns in cirrhotic liver and hepatocellular carcinoma. Int J Cancer 130: 1319-1328, 2012.

9. McKnight R, Nassar A, Cohen C and Siddiqui MT: Arginase-1: a novel immunohistochemical marker of hepatocellular differentiation in fine needle aspiration cytology. Cancer Cytopathol 120: 223-229, 2012.
10. Pan K, Liang XT, Zhang HK, et al: Characterization of BIN1 as a potential tumor suppressor and prognostic marker in hepatocellular carcinoma. Mol Med 18: 507-518, 2012.

11. Andrisani OM, Studach L and Merle P: Gene signatures in hepatocellular carcinoma (HCC). Semin Cancer Biol 21: 4-9, 2011.

12. Qiu J, Huang P, Liu Q, et al: Identification of MACC1 as a novel prognostic marker in hepatocellular carcinoma. J Transl Med 9: 166,2011

13. Inoue H, Mori M, Honda M, et al: The expression of tumorrejection antigen 'MAGE' genes in human gastric carcinoma. Gastroenterology 109: 1522-1525, 1995.

14. Subramanian A, Tamayo P, Mootha VK, et al: Gene set enrichment analysis: a knowledge-based approach for interpreting genome-wide expression profiles. Proc Natl Acad Sci USA 102: 15545-15550, 2005.

15. Yang F, Zhang L, Huo XS, et al: Long noncoding RNA high expression in hepatocellular carcinoma facilitates tumor growth through enhancer of zeste homolog 2 in humans. Hepatology 54: 1679-1689, 2011

16. Yang Z, Zhou L, Wu LM, et al: Overexpression of long non-coding RNA HOTAIR predicts tumor recurrence in hepatocellular carcinoma patients following liver transplantation. Ann Surg Oncol 18: 1243-1250, 2011.

17. Huarte M, Guttman M, Feldser D, et al: A large intergenic noncoding RNA induced by p53 mediates global gene repression in the p53 response. Cell 142: 409-419, 2010.

18. Braconi C, Valeri N, Kogure T, et al: Expression and functional role of a transcribed noncoding RNA with an ultraconserved element in hepatocellular carcinoma. Proc Natl Acad Sci USA 108: 786-791, 2011.

19. Tsai MC, Manor O, Wan Y, et al: Long noncoding RNA as modular scaffold of histone modification complexes. Science 329: 689-693, 2010. 